\title{
Investigation on Recurrent High Dominant Frequency Spatiotemporal Patterns during Persistent Atrial Fibrillation
}

\author{
Xin $\mathrm{Li}^{1}$, Gavin S. Chu ${ }^{2,3}$, Tiago P. Almeida ${ }^{1}$, Frederique J. Vanheusden ${ }^{1}$, Nawshin Dastagir ${ }^{1,2}$, \\ João L. Salinet ${ }^{1,5}$, Peter J Stafford ${ }^{3}$, G. André Ng ${ }^{2,3,4}$, Fernando S. Schlindwein ${ }^{1,4}$ \\ ${ }^{1}$ Department of Engineering, University of Leicester, Leicester, UK \\ ${ }^{2}$ Department of Cardiovascular Sciences, University of Leicester, Leicester, UK \\ ${ }^{3}$ University Hospitals of Leicester NHS Trust, UK \\ ${ }^{4}$ National Institute for Health Research Leicester Cardiovascular Biomedical Research Unit, \\ Glenfield Hospital, UK \\ ${ }^{5}$ Biomedical Engineering, Centre for Engineering, Modelling and Applied Social Sciences, Federal \\ ABC University, Brazil
}

\begin{abstract}
Atrial regions hosting dominant frequency (DF) may represent potential drivers of persistent atrial fibrillation (persAF). Previous work showed that DF can exhibit cyclic behaviour. This study aims to better understand the spatiotemporal behaviours of persAF over longer time periods. 10 patients undergoing persAF ablation targeted at DF were included. Left atrial (LA) non-contact virtual electrograms (VEGMs, Ensite Array, St Jude Medical) were collected for up to 5 min pre-/post-ablation. DF was identified as the peak from $4-10 \mathrm{~Hz}$, in $4 \mathrm{~s}$ windows (50\% overlap). High DF (HDF) map was created and automated pattern recognition algorithm was applied to look for recurring HDF spatial patterns within each patient. Recurring HDF patterns were found in all patients. Patients who changed rhythm to atrial flutter after ablation demonstrated single dominant pattern (DP) among the recorded time period, which might consistent with the higher level of regularity during flutter. Ablation regularized $A F$ as demonstrated by increased DP recurrence after ablation. The time interval (median [IQR]) of DP recurrence for the patients still in atrial fibrillation $(A F)$ after ablation (7 patients) decreased from 21.1 s [11.8 49.7 s] to $15.7 \mathrm{~s}[6.5 \sim 18.2 \mathrm{~s}]$. The proposed method quantifies the spatiotemporal regularity of HDF DPs over long time periods and may offer a more comprehensive dynamic overview of persAF behaviour and the impact of ablation.
\end{abstract}

\section{Introduction}

Atrial fibrillation (AF) is one of the most common arrhythmias in clinical practice, affecting $0.4 \%$ of the general population, and 5\%-10\% of those are over 60 years old $[1,2]$. Although catheter ablation techniques are widely applied to treat AF patients, the underlying pathophysiological mechanisms of long-standing AF remain unclear $[3,4]$. It has shown that atrial sites hosting high dominant frequency (DF) might be the potential important sites for the maintenance of AF [5]. However, recent studies suggest that DF may not be spatiotemporally stable by using noncontact mapping (NCM) in the left atrium (LA). Additionally, we have previously reported the existence of atrial regions with cyclical high DF (HDF) behaviours[6]. In order to further characterise this type of behaviour, an automatic tool has been designed and implemented to investigate the recurrent behaviours of HDF maps over long time periods (up to $5 \mathrm{mins}$ ) to potentially unveil the potential link with spatiotemporal regularity.

\section{Materials and Methods}

\subsection{Data Acquisition}

10 patients undergoing persAF ablation targeted at $\mathrm{DF}$ were included in this study. LA non-contact virtual electrograms (VEGMs, Ensite Array, St Jude Medical) were collected for up to $5 \mathrm{~min}$ pre-/post- ablation for offline analysis.

\subsection{Signal Processing}

The 5-min VEGMs were original sampled at $2034.5 \mathrm{~Hz}$ and then resampled to $512 \mathrm{~Hz}$ to reduce processing time and save storage using cubic interpolation method. Since the ventricular far filed activities affects recorded VEGMs, 


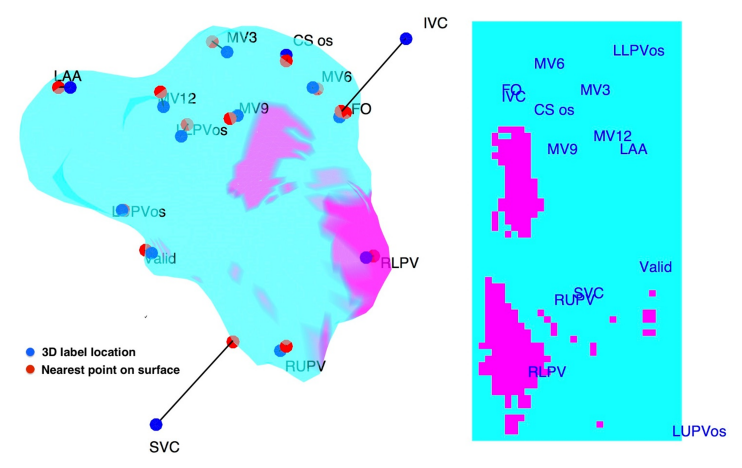

Figure 1. Left: 3D HDF map with location tags and corresponding surface tags ; Right: 2D HDF map with estimated location tags.

QRST subtraction was performed as described in our previous work [7].

The VEGMs were then divided into 4 seconds windows with a $50 \%$ overlap. For each window, spectral analysis was carried out by performing fast Fourier transform with Hamming window and zero padding was used to improve the detection of DF, which was identified as the peak within the physiology range of $4-10 \mathrm{~Hz}$ from the power spectrum. In each window, the nodes were sorted by the DF values in descending order and HDF map was created by the top $10 \%$ of the LA surface area hosting the highest DF. Image binarisation using the HDF threshold was performed using the HDF threshold: the nodes hosting DF values equal or greater than the threshold were transformed to logical value 1 , while the rest of the nodes were labelled with logical value 0 . Equation 1 shows the transformation for the node in row $i$ and column $j$, where $i$ ranges from 1 to the total number of rows, and $j$ ranges from 1 to the total number of columns of the 2D map (Figure 1).

$$
H D F(i, j)= \begin{cases}1, & \text { if } D F(i, j) \geq \text { threshold } \\ 0, & \text { if } D F(i, j)<\text { threshold }\end{cases}
$$

For instance, the 3D and 2D maps in Figure 1 are colourcoded in the HDF fashion after the above image binarisation. Here magenta and cyan stand for 1 and 0 respectively.

\subsection{HDF Pattern Extraction}

$$
c c=\frac{\sum_{i} \sum_{j}\left(A_{i j}-\bar{A}\right)\left(B_{i j}-\bar{B}\right)}{\sqrt{\left(\sum_{i} \sum_{j}\left(A_{i j}-\bar{A}\right)^{2}\right)\left(\sum_{i} \sum_{j}\left(B_{i j}-\bar{B}\right)^{2}\right)}}
$$

The EGM of 2048 virtual electrodes from LA were rearranged on to a $64 \times 322 \mathrm{D}$ uniformed rectangular grid

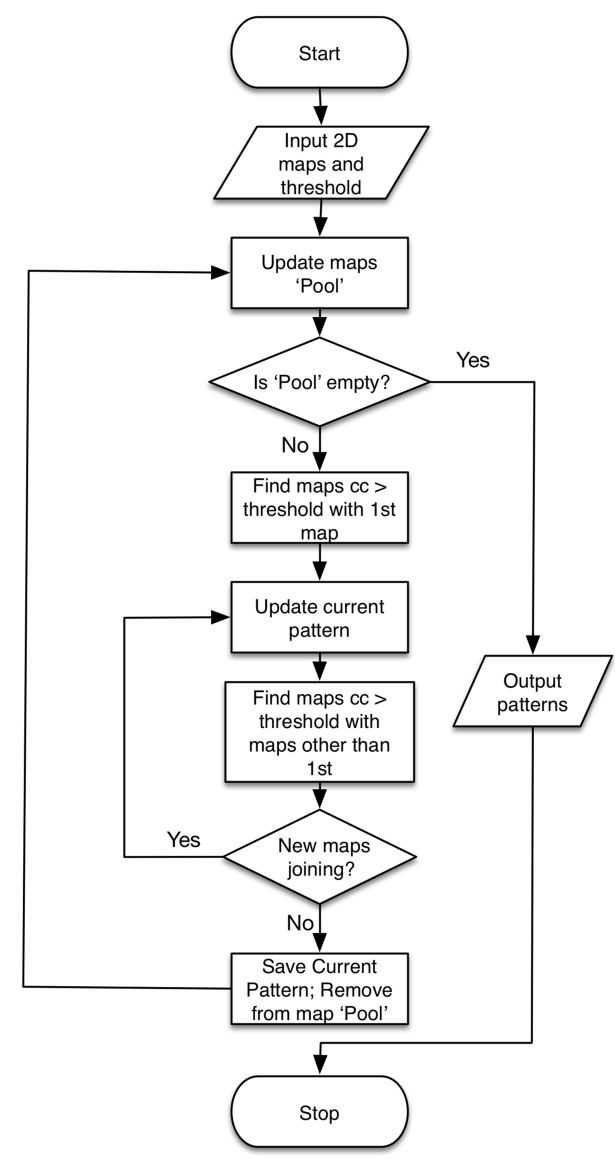

Figure 2. The flowchart of the HDF spatiotemporal pattern extraction algorithm.

(Figure 1 right). In order to find the recurrent HDF maps along time, the pattern extraction algorithm uses 2D Pearson's correlation coefficient (cc) (Equation 2) as a measurement of similarity between the HDF maps (Figure 1) at different time windows [8]. In Equation 2, $A, B$ are 2D images; $\bar{A}, \bar{B}$ are their average values; $i$ and $j$ are the row and column of the images. All the generated HDF maps were included in a data pool, while the single HDF map under analysis was referred as the element. Figure 2 is a flowchart of 2D pattern extraction algorithm using a cc threshold as input. The algorithm can be explained as:

Step 1: Compute the cc of first element of the data pool with the rest, set the elements with cc greater than threshold and the first element as current pattern.

Step 2: Calculate cc of the other elements (if there is any) in current pattern with the elements in the data pool, and add the elements greater than the threshold to the current pattern. Remove the current pattern maps from the data pool, until there are no elements joining the patterns.

Step 3: Consider as a pattern when the element num- 
ber is at least 2 (window recurrence exists) and move on to the next pattern.

Step 4: Repeat Step 1, 2 and 3 until there are no more elements in the data pool, and the patterns were sorted by number of elements and saved.

\subsection{Temporal Analysis}

The above extraction algorithm was applied to the HDF maps from 10 patients prior and after the DF-guided ablation with the $2 \mathrm{D}$ correlation threshold of 0.6. After all the patterns of each patient were found, the dominant pattern (DP) was defined as the pattern with the maximum number of time windows involved, which represents the spatial HDF pattern with more reappearance for each patient(Figure 3A),. The time windows of the DP were recorded in order to investigate the temporal periodic behaviours of the DP HDF maps. The mean time intervals between the windows of the DP and the ratio of duration of the DP windows against the total were calculated. As show in Figure 3B, we visualised the temporal behaviour DP of each patient, the time occurrences of the DP were highlighted as black from the time bar and the other windows shown as grey. In addition, the average time intervals between the DP windows for each patient and the percentage of the duration of DP windows were calculated.

\section{Results and Discussions}

Recurring HDF patterns were found in all patients before and after ablation using cc threshold of 0.6. Figure $3 \mathrm{~A}$ is one example of the recurring pattern of HDF maps in $3 \mathrm{D}$ representation, where the HDF regions repeat in the same or nearby regions with certain level of graphical similarity.

\subsection{Temporal Analysis}

Figure 3B shows the time occurrences of the DP for each patient pre-/post- ablation. Patient 4, 5 and 10 change to atrial flutter (Aflt) after HDF ablation. In these patients, the post-ablation DP accounted for up to $68.1 \%$ (patient 4 ), $94.8 \%$ (patient 5) and $93.6 \%$ (patient 10) of the recorded time period. This finding supports the fact that Aflt can be considered as a more regular arrhythmia, since it is sustained by one macro re-entrance [9]. Therefore, the organized spatiotemporal behaviour of the DP in patients with Aflt after ablation might correlate with the high level of regularity of Aflt.

In general, the time interval (median [IQR]) of DP recurrence for all patients decreased from $16.8 \mathrm{~s}$ [11.5 32.4 s] to $6.5 \mathrm{~s}[3.3 \sim 16.0 \mathrm{~s}]$ after ablation. The time interval (median $[\mathrm{IQR}]$ ) of DP recurrence for the patients still in AF after ablation (7 patients) decreased from $21.1 \mathrm{~s}$ [11.8 49.7

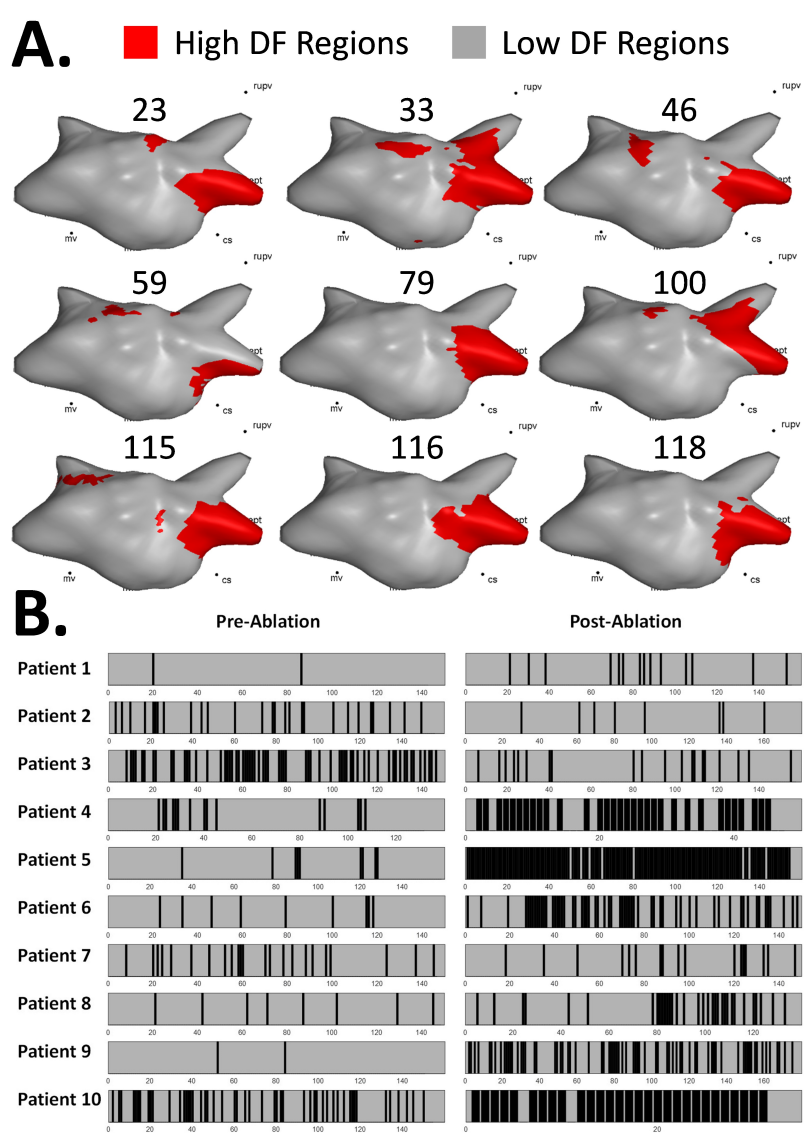

Figure 3. A. 3D LA HDF maps with time window numbers; B. The time occurrences of the DP of each patient pre-/post- ablation (black: DP windows; grey: non-DP windows).

s] to $15.7 \mathrm{~s}[6.5 \sim 18.2 \mathrm{~s}]$. This also might infer the fact that ablating HDF regions might regularize AF demonstrated by increased DP recurrence after ablation.

\subsection{Correlation Coefficient Threshold}

Figure 4 shows the relationship between the fraction of number of time windows in DP over total number of windows and the cc threshold used in the algorithm. As expected, the number of windows reduces when the threshold of the pattern extraction algorithm increases. Further investigation on the threshold balancing the number of DP windows and the quality of the selected HDF maps is crucial, which is the next step of the current study.

\section{Conclusions}

With the technology of non-contact mapping and the proposed spatiotemporal pattern extraction algorithm, we have been able to provide a more dynamic view of the re- 
Table 1. The percentage of duration of DP windows over the recorded time period and the mean interval of recurrences between the DP windows before and after ablation ( ${ }^{1}$ All patients; ${ }^{2}$ Patients still in AF post-ablation).

\begin{tabular}{ccccc}
\hline \multirow{2}{*}{ Patient } & \multicolumn{2}{c}{ DP Duration \% } & \multicolumn{2}{c}{ Mean Interval (s) } \\
\cline { 2 - 5 } & Pre & Post & Pre & Post \\
\hline 1 & 1.4 & 9.0 & 132 & 20.3 \\
2 & 17.4 & 4.9 & 11.2 & 37.1 \\
3 & 43.2 & 12.7 & 4.4 & 15.7 \\
4 & 11.5 & 68.1 & 12.3 & 2.8 \\
5 & 5.7 & 94.8 & 23.3 & 2.1 \\
6 & 6.9 & 40.5 & 21.1 & 5.0 \\
7 & 15.8 & 11.5 & 12.5 & 16.1 \\
8 & 5.4 & 24.6 & 35.4 & 8.0 \\
9 & 1.3 & 7.2 & 64.0 & 4.8 \\
10 & 32.5 & 93.6 & 6.0 & 2.1 \\
\hline Median $^{1}$ & 9.2 & 18.6 & 16.8 & 6.5 \\
{$\left[\right.$ IQR $^{1}$} & {$[5.4 \sim 17]$} & {$[9.6 \sim 61.2]$} & {$[11.5 \sim 32.4]$} & {$[3.3 \sim 16.0]$} \\
Median $^{2}$ & 6.9 & 11.5 & 21.1 & 15.7 \\
[IRQ $^{2}$ & {$[3.4 \sim 16.6]$} & {$[8.1 \sim 18.7]$} & {$[11.8 \sim 49.7]$} & {$[6.5 \sim 18.2]$} \\
\hline
\end{tabular}

current behaviours of DF during AF.

Single recurrent activity found in Aflt might help characterise higher regularization. Reduced recurrent interval of DP after HDF ablation might correspond to the increase in the level of spatiotemporal regularity during persAF. Further study on the features of the HDF regions of recurring patterns may also help to understand the disease.

\section{Acknowledgements}

TPA and JLS have received research grants from Conselho Nacional de Desenvolvimento Científico e Tecnológico (CNPq, Brazil, Grants N. 200251/2012-0; 200598/2009-0, respectively). ND has received financial support from the Leicester National Institute for Health Research Biomedical Research Unit.

\section{References}

[1] Nattel S. Atrial electrophysiology and mechanisms of atrial fibrillation. Journal of cardiovascular pharmacology and therapeutics 2003;8 Suppl 1:S5-S11. ISSN 1074-2484.

[2] Everett TH, Kok LC, Vaughn RH, Moorman JR, Haines DE. Frequency domain algorithm for quantifying atrial fibrillation organization to increase defibrillation efficacy. IEEE Transactions on Biomedical Engineering 2001;48:969-978. ISSN 00189294.

[3] Nattel S. New ideas about atrial fibrillation 50 years on. Nature 2002;415:219-226. ISSN 00280836.

[4] Jalife J, Berenfeld O, Mansour M. Mother rotors and fibrillatory conduction: A mechanism of atrial fibrillation, 2002.

[5] Sanders P, Berenfeld O, Hocini M, Jaïs P, Vaidyanathan R, Hsu LF, Garrigue S, Takahashi Y, Rotter M, Sacher F, Scavée C, Ploutz-Snyder R, Jalife J, Haïssaguerre M. Spectral analysis identifies sites of high-frequency activity maintain-

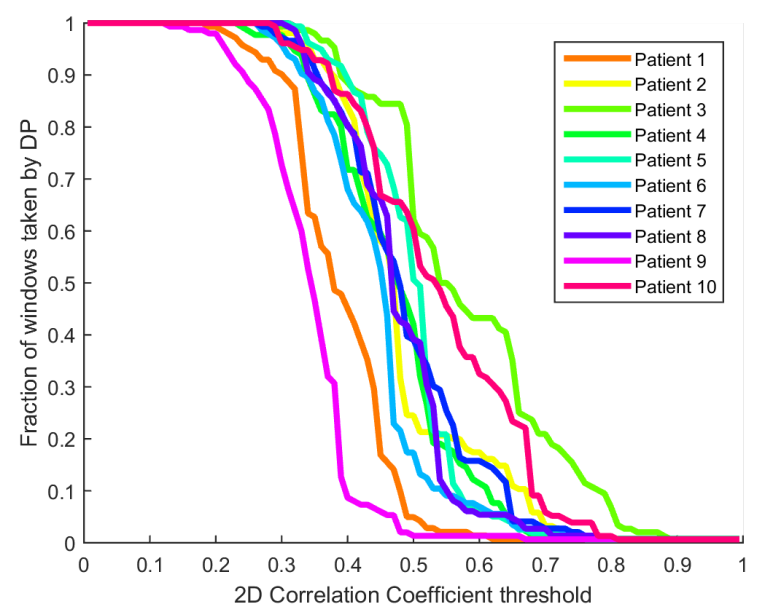

Figure 4. The fraction of number of time windows in DP over total number of windows versus the correlation coefficient threshold applied.

ing atrial fibrillation in humans. Circulation August 2005; 112(6):789-97. ISSN 1524-4539.

[6] Salinet JL, Tuan JH, Sandilands AJ, Stafford PJ, Schlindwein FS, André Ng G. Distinctive patterns of dominant frequency trajectory behavior in drug-refractory persistent atrial fibrillation: preliminary characterization of spatiotemporal instability. Journal of cardiovascular electrophysiology April 2014;25(4):371-9. ISSN 1540-8167.

[7] Salinet JL, Madeiro JPV, Cortez PC, Stafford PJ, André Ng $\mathrm{G}$, Schlindwein FS. Analysis of QRS-T subtraction in unipolar atrial fibrillation electrograms. Medical and Biological Engineering and Computing 2013;51:1381-1391. ISSN 01400118.

[8] Pearson K. Mathematical contributions to the theory of evolution. iii. regression, heredity, and panmixia. Philosophical Transactions of the Royal Society of London Series A Containing Papers of a Mathematical or Physical Character 1896; 187:pp. 253-318. ISSN 02643952.

[9] Winter JB, Crijns HJ. Atrial flutter and atrial fibrillation: two sides of a coin or one coin? Journal of cardiovascular electrophysiology 2000;11(8):859-860. ISSN 10453873.

Address for correspondence:

Xin Li

Department of Engineering

University of Leicester

LE1 7RH

UK

x1153@le.ac.uk

leexin0121@gmail.com 